\title{
A study on lightning protection system - the effect of bending degree of the down conductor
}

\begin{abstract}
Lightning is one of nature's most powerful and destructive phenomena. Lightning discharges contain awesome amounts of electrical energy and even though a lightning discharge is of a very short duration, but it is a very real cause of damage and destruction. Thus, nothing may exclude from being strike by the lightning but lightning protection system could give the reliable protection via its good installation. The major role of lightning protection is to secure a structure from lightning damage by intercepting flashes and then conduct the currents to the ground quickly and safely where its main components are the air termination network, the down conductor and the earth termination. This paper will focus on the effect of bending the down conductor on the electromagnetic of the current flow in the down conductor. Therefore, the main objectives are to investigate and analyses the effect of bending degree of down conductor to the electromagnetic of current flow through the down conductor and to ensure the path for the down conductor to discharge the lightning current to the ground is of low impedance and reduce the probability of damage due to the high impulse lightning current. The overall findings are concluded and discussions are made to explain the overall simulation result at the end of project.
\end{abstract}

Keyword: Down conductor; Bending; Lightning strike; Electromagnetic 